\title{
СУБОПТИМАЛЬНЫЙ СИНТЕЗ РЕГУЛЯТОРОВ МНОГОМЕРНЫХ ЛИНЕЙНЫХ СИСТЕМ СПОСОБОМ АГРЕГАЦИИ
}

\author{
(Представил Н. Алумяэ)
}

Рассматривается задача управления многомерной линейной системой с квадратичным функционалом оптимальности. Исследуется вопрос определения матриц усиления, arpeгации и приближенных регуляторов, субоптимальных по показателям типа КлейнманаАтанса и Ульма. С этой целью применяются три критерия субоптимальности:

1) близости приближенного регулятора к оптимальному по евклидовой норме невязки эффективной матрицы;

2) математического ожидания исходного критерия;

3) равномерно-фазового и фазового показателя субоптимальности.

Получены необходимые условия экстремальностн матриц по этим критериям и предложен синтез линейных субоптимальных регуляторов, обобщающий результаты работ $\left[{ }^{1-7}\right]$.

\section{1. Постановка задачи}

Рассмотрим задачу оптимизации многомерной $(n \gg 1)$ линейной системы

$$
x=A(t) x+B(t) u, \quad \operatorname{dim} x=n \geqslant r=\operatorname{dim} u=r_{B} \triangleq \operatorname{rank} B
$$

по функционалу

$$
2 J=x_{1}^{\prime} P_{1} x_{1}+\int_{t_{0}}^{t_{1}}\left(x^{\prime}(t) Q(t) x(t)+u^{\prime}(t) R(t) u(t)\right) d t
$$

где $x, u-$ векторы состояния и управления, $u(t) \in C[\mathcal{G}], \quad \mathcal{C}=\left[t_{0}, t_{1}\right]$, матрицы $P_{1}=P\left(t_{1}\right)=$ const, $A(t), B(t), Q(t), R(t) \in C[\mathcal{G}]$ нужного порядка

$$
Q=Q^{\prime} \geqslant 0, \quad R=R^{\prime}>0, \quad P_{1}=P_{1}^{\prime} \geqslant 0
$$

и фиксированы $t_{0}, t_{1}, x_{0} \triangleq x\left[t_{0}\right]=$ const. При условиях $(1.1),(1.2)$ по $x$ существует линейный, единственный оптимальный $u=u^{0}(t, x)$ регулятор

$$
u^{0}=K^{0}(t) x, \quad{ }_{r} K^{0 n}=-R^{-1} B^{\prime} M, \quad M={ }_{n} M^{n}(t), \quad B^{\prime} \triangleq B^{\mathrm{T}},
$$

где матрицант $M(t)$ - решение уравнения Риккати $\left[{ }^{8,9}\right]$

$$
M \cdot=M B R^{-1} B^{\prime} M-A^{\prime} M-M A-Q,\left.\quad M\right|_{t=t_{1}} \stackrel{\triangle}{=} M_{1}=P_{1} \geqslant 0 .
$$

Решение (1.4) существует, единственно, $M=M^{\prime}$ на $\mathfrak{C}$, положительно определенная на $\mathcal{G} \backslash t_{1}: M>0$. Минимум $2 J$ по $u(t)$ равен $2 J^{0}\left(t_{0}, x_{0}\right)=$ $=x_{0}{ }^{\prime} M\left(t_{0}\right) x_{0}$. Реализация решения (1.4) предъявляет большие требования к памяти ЭВМ, где надо сохранять значения ${ }_{n} M^{n}\left(t_{i}\right)$ или ${ }_{r} K^{n}\left(t_{i}\right)$. 
Вычисление и измерение $x\left(t_{i}\right)$ должны быть много быстрее эволюции $(1.1)$, а ошибка, пропорциональная объему счета. значительна при $n \gg 1$. Первое из ограничений вычислительного характера. казалось бы. устраняется интеграцией (1.4) справа налево с вычислением $M\left[t_{0}\right]$. Интеграпией (1.4) в прямом времени, находим в ходе процесса управления $M\left(t_{i}\right)$. Но из-за неустойчивости этого процесса вычисления мы лишены такой возможности $\left[{ }^{6}\right]$.

Реально часто есть ограничение в информации об $x$, когда измеряется лишь сигнал $z=C x$, где ${ }_{l} C^{n} \in C[\mathcal{G}]-$ матрица ранга $r_{r}=l$ $(l \ll n, l \leqslant n)$. (Дифференцирование $z$-компоненты $x$ для наблютения $x$ по $(1.1),(1.3)$ и $C . d^{k} z / d t^{k}(0 \leqslant k \leqslant n-1)$ сопряжено с ростом погрешностей вычисления и техническими трулностями [9].) Учитывая эти условия, будем на основе линейной агрегации $\left.{ }^{4-7}\right\rceil$ осуществлять приближенный синтез $\bar{u}$, опрелеленный одним из трех вариантов субоптимизации по критериям $[1,2,4,6,7]$.

\section{2. Минимизация квадратичной меры отклонения $\bar{u}$ от $u^{0}$}

Учитывая линейность $(1.1),(1.3)$ и сигнала $z$ по $x$, будем искать приближенный регулятор $\bar{u}$ (и соответствующий $I$-субоптимальный $\left.\bar{u}_{0}\right)$ в классе $\bar{u}=\bar{K} C x$, где $r_{c}=l, C[\mathcal{C}] \in{ }_{r} \bar{K}^{l},{ }_{l} C^{n}-$ матрицы усиления и агрегации соответственно. Идеально найти $C, \bar{K}$ из $\bar{K} C=K^{\circ}$, но так как rank $K^{0}=r$ и рассматривается сильная агрегация $r_{c}=l<r$, то уравнение не имеет решения. Поэтому для $\forall t \in \mathcal{G}$ рассмотрим задачу минимизации по $C, \bar{K}$ квадрата нормы невязки.

$$
I=\left\|\bar{K} C-K^{0}\right\|_{(2)}^{2}\left(\|A\|_{(2)}^{2} \triangleq \operatorname{tr}\left(A A^{\prime}\right), K^{0}=-R^{-1} B^{\prime} M\right)
$$

Из $\delta I=0$ аналогично $\left[{ }^{6}\right]$ находим необходимые условия (2.1)-экстремальности $C_{0}, \bar{K}_{0}$ в виде двух матричных уравнений

$$
{ }_{1}^{1} / 2 \operatorname{grad}_{C_{0}} I=\bar{K}_{0}^{\prime}\left(\bar{K}_{0} C_{0}-K^{0}\right)={ }_{\imath} 0^{n}, \quad 1 / 2 \operatorname{grad}_{\bar{K}_{0}^{\prime}} I=C_{0}\left(C_{0}^{\prime} \bar{K}_{0}^{\prime}-K^{0^{\prime}}\right)={ }_{l} 0^{r} .
$$

Второе из них удовлетворяется лишь при

$$
\bar{K}_{0}=K^{0} C_{0}^{\prime}\left(C_{0} C_{0}^{\prime}\right)^{-1} \quad\left(r_{c_{0}}=l \Leftrightarrow \Delta\left(C_{0} C_{0}^{\prime}\right)>0\right) .
$$

Из (2.2), (2.3) находим все экстремальные «эффективные» матрнцы $\widetilde{K}_{0}$

$$
\widetilde{K}_{0}=\bar{K}_{0} C_{0}=P\left[\Theta^{(1)^{\prime}}\right] K^{0}, \quad P\left[\Theta^{(1)^{\prime}}\right] \stackrel{\triangle}{=} P^{\prime} P, \quad P P^{\prime}={ }_{l} \mathbf{1}^{l}, \quad P=(l \times r),
$$

где $(r \times r)$ - матрица проектирования $P\left[\Theta^{(1)^{\prime}}\right]$ перестановочна с $\left(K^{0} K^{0}\right)$ ранга $r$, т. е. $\left.P\left[\Theta^{(1)}\right]=\Theta \digamma 0_{\rho}, 1_{i}\right\lrcorner, \Theta=\left[\Theta^{(1)} \mid \Theta^{(2)}\right], \Theta^{(1)}=(r \times \varrho)$. $\Theta^{(2)}=(r \times l)$ - субматрицы ортогональной $\Theta$ из собственных векторов $\left(K^{0}, K^{0}\right)$, соотвегствующих еe собственным числам $k_{s}{ }^{2}$ в порядке их

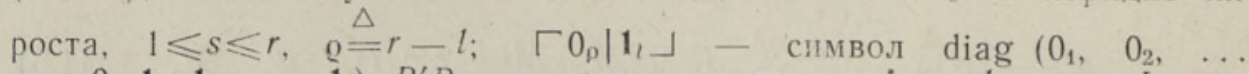
$\left.\ldots, 0_{l}, \mathbf{1}_{1}, \mathbf{1}_{2}, \ldots, \mathbf{1}_{l}\right), P^{\prime} P-$ матрица проектированіия в $l$-мерное $L$ подпространство $r$-мерных столбцов $\Theta^{(2)}$, а $\Theta^{(1)^{\prime}} \Theta^{(2)}=0$. Если $l$ максимальньіх чнсел спектра $\left(K^{0} K^{\prime}\right)$ - различны, то плоскость $L-$ единстветная. Стац!онарное значение $\rho_{\Sigma} k_{s}^{2}=I \searrow 0$ при $l \rightarrow r$. Из $(2.2)-(2.4)$ на $C_{0}$ следует уравнение

$$
\begin{gathered}
C_{0}^{\prime}\left(C_{0} C_{0}^{\prime}\right)^{-1} C_{0}=K^{0^{\prime}} P^{\prime} P\left(K^{0} K^{0^{\prime}}\right)^{-1} P^{\prime} P K^{0}+P\left(K^{0}\right) W P\left(K^{0}\right), \\
P\left(K^{0}\right)={ }_{n} 1^{n}-K^{0 \prime}\left(K^{0} K^{0 \prime}\right)^{-1} K^{0}
\end{gathered}
$$


с произвольной симметричной $(n \times n)$-матрицей $W$. Решением $(2.5)$ яв ляется $C_{0}=V_{\omega(2)}, r\left(C_{0}\right)=l$, где ${ }_{l} V^{l}-$ неособая произвольная такая, что $r_{C_{0}}=l$, а $\omega_{(2)}$ - субматрица $(l \times n)$ ортогональной $\omega=(r \times n)$, представляющей $K^{0}=\Theta\left\ulcorner k_{1}, \ldots, k_{r}\right\lrcorner \omega, \omega^{\prime} \triangleq\left[\omega_{(1)}^{\prime} \mid \omega_{(2)}^{\prime}\right]$. Субоптимальное $\bar{u}=$ $=\bar{K}_{0} x$ по $I$ дает проектирование: надо оптимальный вектор $K^{0} x$ спроекгировать на $l$-мерную $L \in E^{r}$. В интеграции (1.4) нужно на каждом шаге иметь матрицу $\left(P^{\prime} P\right)$ перестановочную с $\left(K^{0} K^{\nu^{\prime}}\right)$, но значения $P$, $K_{0}, M$ не надо сохранять в памяти $Э В М$, так как они сразу используются при вычислении $\bar{u}=\widetilde{K}_{0} x=\bar{K}_{0}\left(C_{0} x\right)$. В отличие от $\left[{ }^{6}\right]$ здесь $C$ не задана и $C=C[t] \not \equiv$ const, а условия $(2.2)$ охватывают задачи $\min I$ по $\bar{K}(C=$ fix $)$ и $\min I$ по $C(K=\mathrm{fix})$. В силу $(2.3)-(2.5)$ экстремальная $\widetilde{K}_{0}$ ранга $l$.

\section{3. Минимизация вероятностного критерия типа Клеймана-Атанса}

Значение $2 J(t, x)$ при $(1.1)$ и $u=\widetilde{K} x$ будет квадрикой $2 \bar{J}=x^{\prime} L(t) x$, где матрица $L\left[t, t_{1}, L_{1} ; \tilde{K}\right]$ удовлетворяет линейному уравнению

$$
\begin{gathered}
-L=L \tilde{A}+\widetilde{A}^{\prime} L+\widetilde{Q}, \quad L\left(t_{1}\right)=L_{1}=P_{1}, \quad \widetilde{A}=A+B \widetilde{K}, \\
\widetilde{Q}=Q+\widetilde{K}^{\prime} R \widetilde{K}, \quad \widetilde{K} \triangleq \bar{K} C,
\end{gathered}
$$

имеющему на $\mathscr{C}$ единственное, положительно определенное вне $t_{1}$ решение $L^{\prime}=L>M$ (в силу единственности $u^{0}$ ) вида

$$
L=\Phi^{\prime}\left(t_{1}, t\right) P_{1} \Phi\left(t_{1}, t\right)+\int_{t}^{t_{1}} \Phi^{\prime}(\tau, t) \widetilde{Q}(\tau) \Phi(\tau, t) d \tau .
$$

Фундаментальная $(n \times n)$-матрица $\Phi[t, \tau ; \tilde{A}]$ удовлетворяет равенствам

$$
\begin{gathered}
\Phi^{*}=\tilde{A} \Phi, \quad \Phi(\tau, \tau ; \cdot)={ }_{n} \mathbf{1}^{n}, \quad \Phi\left(t_{1}, t_{3}\right)=\Phi\left(t_{1}, t_{2}\right) \Phi\left(t_{2}, t_{3}\right), \\
\Phi(t, \tau)=\Phi(t, 0) \Phi^{-1}(\tau, 0) .
\end{gathered}
$$

Положим $N_{0}=L_{0}-M_{0}, F_{0} \triangleq F \mid t=t_{0}$ и рассмотрим разность

$$
2 \Delta J^{0}=2\left[\bar{J}\left(t_{0}, x_{0}\right)-J^{0}\left(t_{0}, x_{0}\right)\right]=x_{0}^{\prime} N_{0} x_{0}, \quad N_{0}>0,
$$

для которой в разделе 4 найдем равномерные и точечные по $x_{0}$ условия минимизации $\Delta J^{0}$ по $C, \bar{K} \in C[\mathcal{G}]$. Не проводя здесь эту минимизацию, рассмотрим

$$
\Delta_{i}=\mu_{i}^{-1}, \quad d_{i}=\lambda_{i}^{-1}, \quad G\left(M_{0}\right)=\left\{x_{0} \mid x_{0}^{\prime} M_{0} x_{0} \leqslant 1\right\}, \quad G\left(L_{0}\right)=\left\{\bar{x}_{0} \mid \bar{x}_{0}^{\prime} L_{0} \bar{x}_{0} \leqslant 1\right\} .
$$

Длины их полуосей $\Delta_{i}=\mu_{i}^{-1}, d_{i}=\lambda_{i}^{-1}$, где $\mu_{i}, \lambda_{i} \quad$ - корни из чисел спектров $M_{0}, L_{0}$ в порядке их роста, $d_{n} \leqslant \Delta_{n}, d_{1} \leqslant \Delta_{1}(1 \leqslant i \leqslant n)$.

В выборе критерия субоптимальности $\bar{u}$ играют роль три мотива:

1) этот критерий должен давать удовлетворительную оценку $\Delta J^{0}$,

2) ему отвечает эффективная процедура решения задачи или субоптимальное решение ее в аналитической форме,

3) критерий субоптимальности имеет геометрический смысл. Учитывая $(3.1)-(3.5)$ и условия 1)-3), положим $\quad|| N_{0} \| \triangleq\left|\operatorname{tr} N_{0}\right|$ удовлетворяющей свойствам нормы $N_{0}$. На множестве $\bar{K}, C \in C[\mathcal{G}]$ поставим задачу минимизировать $\left\|N_{0}\right\|$, эквивалентную двум задачам минимизации: близости $\bar{J}$ к $J^{0}$ в среднем на $\left\|x_{0}\right\| \leqslant 1$

$$
2\left\|\Delta J^{0}\right\|_{\left(L_{t}\right)}=\int_{\left\|x_{0}\right\| \leqslant 1}\left|x_{0}^{\prime} N_{0} x_{0}\right| d x_{0}=c(n) \operatorname{tr} N_{0} \quad(c(n)=\text { const })
$$


и математического ожидания, если $P\left(x_{0}\right)$ - непрерывная функция лишь $r_{0}$

$$
r_{0}=\left(x_{0}^{\prime} x_{0}\right)^{1 / 2} \text { при } P \leqslant C^{0} r_{0}^{(3+\varepsilon)} \text { вне } R^{0}=\text { const }\left(r_{0}>R^{0}, 0<\varepsilon\right)
$$

$$
E\left[\Delta^{0}\right]=1 / 2 \int_{\|x\|<\infty}\left(x_{0}^{\prime} N_{0} x_{0}\right) P\left(r_{0}\right) d x_{0}=c(n ; P) \operatorname{tr} N_{0}\left(c^{0}, c(n ; P)=\text { const, } \varepsilon>0\right) .
$$

Смысл этих задач - минимизация по $\bar{K}, C$ разности сумм диаметров (3.5). Ввиду аддитивности $\operatorname{tr}$ это эквивалентно минимизации меры

$$
I_{1}=\left\|L_{0}\right\|=\operatorname{tr} L\left[t_{0}, t_{1}, L_{1} ; \widetilde{K}\right] \quad \text { на } \bar{K}, C \in C[\mathcal{C}], \widetilde{K} \triangleq \bar{K} C,
$$

не содержащей $M_{0}$. Остальные инварианты вращения $I_{2}, \ldots, I_{n} \triangleq \operatorname{det} L_{0}$ для $L_{0}$ - возможные меры близости $G\left(L_{0}\right)$ к $G\left(M_{0}\right)$. При минимизацин разности их объемов минимизируем : $\operatorname{det} L_{0} \triangleq \Delta\left(L_{0}\right)\left[{ }^{7}\right]$, но $I_{j}\left(L_{0}\right)$ неаддитивные и не имеют всех свойств нормы $\bar{L}_{0}, 2 \leqslant j \leqslant n$. Рассмотрим задачу Майера с критерием (3.6) при связях (3.1) и $C, \bar{K} \in C[\mathcal{G}$, где надо найти экстремальные $C^{0}, \bar{K}^{0}$, решающие задачу $\operatorname{Arg} \min I_{1}$ при $\nabla t \in \mathcal{C}$ и существовании их. Введем варьированные матрицы и обозначения

$$
\begin{gathered}
C_{\varepsilon}=C^{0}+\varepsilon V, \quad \bar{K}_{\varepsilon}=\bar{K}^{0}+\varepsilon U\left({ }_{l} V^{n},{ }_{r} U^{l} \in C[\mathcal{C}], K_{0} \triangleq-R{ }^{-1} B^{\prime} L\right), \\
\widetilde{K}_{\varepsilon}=\widetilde{K}^{0}+\varepsilon \widetilde{K}_{1}+0(\varepsilon) \widetilde{K}_{2}(\cdot, \varepsilon), \widetilde{K}^{0} \stackrel{\triangle}{=} \bar{K}^{0} C^{0}, \quad \widetilde{K}_{1} \triangleq \bar{K}^{0} V+U C^{0}, \quad S^{\prime} S \stackrel{\triangle}{=} R, \\
\Psi=\Psi^{\prime} \triangleq \Phi\left(t, t_{1}, \cdot\right) \Phi^{\prime}\left(t, t_{1}, \cdot\right)>0, \quad H\left(\widetilde{K}^{0}\right) \stackrel{\triangle}{=} \Psi\left[B^{\prime} L+R \bar{K}^{0} C^{0}\right]^{\prime}=(n \times r) .
\end{gathered}
$$

Используя (3.1)-(3.3), (3.7), элементарные свойства tr, из первого приближения по $\varepsilon$ получим вариацию $\delta I_{1}=\left(\partial I_{1} / \partial \varepsilon\right) \mid \varepsilon=0$ вида

$$
1 / 2 \delta I_{1}=\int_{t_{0}}^{t_{1}} \operatorname{tr}\left[H \bar{K}^{0} V+C^{0} H U\right] d t .
$$

Необходимые условия экстремальности $C^{0}, \bar{K}^{0}$ по (3.6) будут

$$
1 / 2 \operatorname{grad}_{c^{0}} I_{1}=\bar{K}^{0 \prime} H^{\prime}={ }_{l} 0^{n}, \quad{ }^{1} / 2 \operatorname{grad}_{\bar{K}^{0}} I_{1}=H^{\prime} C^{0}={ }_{r} 0^{l}
$$

с учетом (3.7) эквивалентны нелинейным по $C^{0}, \bar{K}^{0}$ уравнениям

$$
\left(L B+C^{0^{\prime}} \bar{K}^{0 \prime} R\right) \bar{K}^{0}={ }_{n} 0^{l}, \quad C^{0} \Psi\left(L B+C^{0 \prime} \bar{K}^{0 \prime} R\right)={ }_{l} 0^{r} .
$$

Они сложнее, но по структуре аналогичны условиям экстремальности $C_{0}, \bar{K}_{0}, \bar{K}_{0}$ по $I$. Действительно, преобразованием $\quad C^{0}=C_{0} \Phi, \bar{K}^{0}=S \bar{K}_{0}$ $K_{0}=S K^{0} \Phi$ система (3.8) сведется к (2.2). Используя результаты $(2.3)-(2.5)$, находим определяющую экстремальный по $I_{1}$ субоптимальный регулятор $\bar{u}=\widetilde{K}^{0} x$ и эффективную матрицу

$$
\widetilde{K}^{0}=\bar{K}^{0} C^{0}=S^{-1}\left(P_{0}^{\prime} P_{0}\right) S K_{0}, \quad P_{0} P_{0}^{\prime}={ }_{l} 1^{l} \quad\left(P_{0}^{\prime} P_{0} \unrhd_{r} \mathscr{T}_{0}^{r}, r\left(T_{0}\right)=l\right),
$$

где проектор $\mathfrak{T}_{0}$ необходимо перестановочен с $S K_{0} \Psi K_{0}{ }^{\prime} S^{\prime}$ ранга $r$. Если $\Theta_{0}$ - диагонализирующая матрица последней, то это условие эквивалентно

$$
\left.\Im_{0}=\Theta_{0} \Gamma 0_{\rho}, \mathbf{1}_{l}\right\lrcorner \Theta_{0}^{\prime}\left(\Theta_{0} \Theta_{0}^{\prime}={ }_{r} \mathbf{1}^{r}, \varrho \triangleq r-l\right) .
$$

Из (3.9) аналогично разделу 2 следует, что для синтеза регулятора 
$\widehat{K}^{0} x$ надо на каждом шаге совместно решать $2(n \times n)$-мерную линейнуюิ задачу (3.1)-(3.3) Коши и алгебраическую - поиска $\Theta_{0}$ для $S K_{0} \Psi K_{0}^{\prime} S^{\prime}$. Значения $\widetilde{K}^{0}$ не обязательно сохранять в памяти ЭВМ. Аналогично разделу 2 при переходе $l \rightarrow l+1$ показатель $I_{!}$не возрастает, т. К. $r \bar{K}^{0 l},{ }_{l} C^{0} n-$ соответственно субматрицы для $r \bar{K}^{l+1}, C_{i+1}^{n}$. В рассматриваемых вариантах не используются обычные для задачи Майера множители Лагранжа, что упрощает синтез $\bar{u}$. В отличие от локальной схемы агрегирования, основанной на теории возмущений с малым параметром $\varepsilon\left[{ }^{10}\right]$, предлағаемые вариаиты субоптимизации определяются условиями стационарности рассматриваемых функционалов. Точечно опгимальный регулятор (1.3) имеет матрицу $K=K^{0}=-R^{-1} B^{\prime} M$, являющуюся единственной оптимальной в задаче $\min I_{1}$ по $K$, где $L$ определена (3.1), $\quad \widetilde{K} \rightarrow K \neq \bar{K} C, I_{1} \triangleq \operatorname{tr} L_{0}$. Действительно, решая ее с учегом произвольности $K_{1}-$ вариации оптимальной $K_{*}$ аналогично разделу 3 находим, что (3.8) вырождается в $H\left(K_{*}\right)={ }_{n} 0^{r}$, т. е. $K_{*}=$ $=-R^{-1} B^{\prime} L$. Но тогда $L$ удовлетворяет $(1.4)$ и $L(t) \equiv M(t)$. Поэтому точечно оптимальный $u^{0}$ оптимален также по $I_{1}$, т. е. «в среднем». Из единственности $u^{0}$ следует $L_{0}[\cdot, K]>M_{0}\left[\cdot, K^{0}\right], \operatorname{tr} L_{0}[\cdot, K]>\operatorname{tr} M_{0}\left[\cdot, K^{0}\right]$, т. е. $I_{1}$ имеет в $K^{0} \not \equiv K$ строгий минимум. Так как соответствующие показателям $J$ и $I_{1}$ экстремальные регуляторы $u^{0}$ и $u_{*}=K_{*} x$ совпадают, то функционалы $J$ и $I_{1}$ являются конэкстремальными.

\section{4. Вариант равномерно-фазовой и точечной субоптимизации}

Найдем необходимые условия экстремальности пар $C_{*}, \bar{K}_{*} \in C[\mathcal{G}]$ по функционалу $\bar{J}[K]$ для всех $x_{0} \in R^{n}$ (равномерно-фазовый случай) или для $\forall x_{0} \in R^{n}$ в случае точечной субоптимизации в предположении их существования. Используя (3.1)-(3.3), рассмотрим первую задачу. Введем обозначения

$$
L_{1}=\delta L_{\star}=\left(\partial L_{\varepsilon} / \partial \varepsilon\right)_{\varepsilon=0}=\partial L\left[\widetilde{K}_{*}+\varepsilon(\cdot) ; \cdot\right] / \partial \varepsilon \mid \varepsilon=0,
$$

где $\left.\quad L_{11} \triangleq L_{1}\right|_{t=t_{1}}=0, \quad-L_{1}=L_{1} \bar{A}+\bar{A}^{\prime} L_{1}+\bar{Q}_{1}, \quad \bar{K}_{*} \triangleq \bar{K}_{*} \mathrm{C}_{*}, \quad \bar{A} \triangleq A+B \bar{K}_{*}$,

$$
\begin{gathered}
\left.L_{10} \triangleq L_{1}\right|_{t=t_{0}}=\int_{t_{0}}^{t_{1}} \Phi^{\prime}\left(\tau, t_{0}\right) \bar{Q}_{1}(\tau) \Phi\left(\tau, t_{0}\right) d \tau, \quad \bar{Q}_{1}=\Omega_{1}[U ; t]+\Omega_{2}[V ; t], \\
\Omega_{1} \triangleq D \bar{K}_{*} U+U^{\prime} \bar{K}_{*}^{\prime} D^{\prime}, \quad \Omega_{2} \triangleq D V C_{*}+C_{*}^{\prime} V^{\prime} D^{\prime}, \quad D \triangleq L B+\bar{K}_{*}^{\prime} R, \\
{ }_{l} U^{n} \stackrel{\triangle}{=} \delta C_{*}, \quad{ }_{r} V^{l}=\delta \bar{K}_{*} \in C[\mathcal{C}] .
\end{gathered}
$$

Из (4.1) и условия экстремальности $\bar{J}[\widetilde{K}]$ на $\widetilde{K}$ * для всех $x_{0}$

$$
2 \delta \bar{J}=x_{0}^{\prime} L_{10} x_{0}=0 \sim L_{10}=-L_{10}^{\prime} \Rightarrow L_{10}=0 \text {, так как } L_{10}=L_{10}^{\prime}
$$

имеем при $\forall U, V \in C[\mathcal{G}]$ условия стационарности $C_{*}, \bar{K}_{*}$ вида

$$
\begin{gathered}
\Omega_{1}=\Omega_{1}^{\prime}=D \bar{K}_{*} U+U^{\prime} \bar{K}_{*}^{\prime} D^{\prime}={ }_{r} 0^{n} \quad\left(D \triangleq L B+C_{*}^{\prime} \bar{K}_{*}^{\prime} R\right), \\
\Omega_{2}=\Omega_{2}^{\prime}=D V C_{*}+C_{*}^{\prime} V^{\prime} D^{\prime}={ }_{n} 0^{n} .
\end{gathered}
$$

Подставляя $U=\bar{K}_{*}^{\prime} D^{\prime}$ в $(4.2)$, находим, что оно эквивалентно нелинейному матричному уравнению на $C_{*}, \bar{K}_{*}$ вида

$$
D \bar{K}_{\star}=\left[L B+\left(\bar{K}_{*} C_{*}\right)^{\prime} R\right] \bar{K}_{*}={ }_{n} 0^{l} .
$$


ПОдстановкой $\cdot \dot{V}^{0}=D^{\prime} C_{*}^{\prime}\left(C_{*} C_{*}^{\prime}\right)^{-1}$ в (4.3) находим условие

$$
C_{*} D \triangleq C_{*}\left(L B+\widetilde{K}_{*}^{\prime} R\right)=l 0^{r} . \quad\left(\operatorname{rank} C=r_{C}=l\right) .
$$

Умножая (4.3) на $C_{\star}{ }^{\prime}$ справа, из (4.5) находим второе требование, определяющее вместе с (4.4) равномерно-фазовый случай

$$
D V=\left(L B+C_{*}^{\prime} \bar{K}_{*}^{\prime} R\right) V={ }_{n} 0^{l} .
$$

В нем ранг матрицы $V \triangleq \delta K_{\star}$ возможных вариаций есть $d=r-r_{D}$. Легко показать, что $V^{0}$ входит в (4.6) лишь при условии (4.5), которое в общем случае не будет необходимым и достаточным для (4.3). Рассмотрим $\operatorname{rank} D$ в подслучае (4.4), (4.5), когда $V^{0} \in\{V\}$. Заменой $C_{\star} \rightarrow C_{0}, \bar{K}_{*} \rightarrow R^{-1 / 2} \bar{K}_{0}, L B \rightarrow M B R^{-1 / 2}$ сводим (4.4), (4.6) к (2.2). Используя результаты $(2.3)-(2.5)$, получим

$$
\widetilde{K}_{*}=\bar{K}_{*} C_{*}=-\tilde{\Psi} B^{\prime} L, \quad D=L B R^{-1}\left[1_{r}^{r}-R^{1 / 2}\left(P_{*}^{\prime} P_{*}\right) R^{-1 / 2}\right], \quad r_{D}=r-l,
$$

где $R^{-1 / 2} B^{\prime} L^{2} B R^{-1 / 2}$ необходимо перестановочна с $P_{*}^{\prime} P_{*}, P_{*} P^{\prime}={ }_{l} 1^{l}$, $\tilde{\Psi} \triangleq R^{-1 / 2}\left(P_{*}^{\prime} P_{*}\right) R^{-1 / 2}$. При $\widetilde{W} \triangleq R^{-1 / 2} V$ уравнение (4.6) сводится к $\left[r^{r}-P_{*}^{\prime} P_{*}\right] W={ }_{r} 0^{l}, \quad$ а решение (4.6) при (4.5) будет $R^{1 / 2} P_{*}^{\prime} w$, где $w=(l \times l)-$ произвольная, $w \in C[\mho]$. В общем случае $(4.4),(4.6)$ обозначим

$$
\begin{gathered}
{ }_{r} Y^{l} \triangleq R^{1 / 2} \bar{K}_{*}, \quad{ }_{n} N^{r}=L B R^{-1 / 2}, \quad M \triangleq L B, \quad G=D R^{-1 / 2} \quad\left(r_{N}=r\right), \\
D \bar{K}_{\star}=\left(N+C_{*}^{\prime} Y^{\prime}\right) Y=G Y={ }_{n} 0^{l}, \quad r_{G}=r_{D} .
\end{gathered}
$$

С учетом (4.7) можно доказать эквивалентность (4.4) уравнению на $Y$

$\left(H H^{\prime} N\right) Y={ }_{n} 0^{l}, \quad H H^{\prime}={ }_{n} 1^{n}-C_{*}^{\prime}\left(C_{*} C_{*}^{\prime}\right) C_{\star}=P_{C_{*}}, \quad H^{\prime} H=1_{n-l}^{n-l}$,

где

$$
\operatorname{rank} D=\operatorname{rank}\left(H H^{\prime} N\right)=\operatorname{rank}\left(P_{C_{*}} N\right)=\operatorname{rank} P_{C_{*}} M .
$$

В общем -случае $(4.4),(4.6)$ из (4.7), (4.8) заключаем, что экстремальная пара $C_{*}, \bar{K}_{*}$ первой задачи связана (4.4) при линейном условии (4.6) на $\delta \bar{K}_{*}=V$, где rank $D=\operatorname{rank}\left(P_{C}, L B\right)$,

$$
0 \leqslant r_{V} \triangleq d=r-\operatorname{rank}\left(P_{C}, L B\right) \geqslant r-\min \{r, n-l\} .
$$

Рассмотрим задачу точечной субоптимизации, используя (4.1). Из $\delta \bar{J}=0$ при $\forall U, V \in C[\mathcal{G}]$ аналогично $(4.2),(4.3)$, находим на $C_{*}, \bar{K}_{\star}$ необходимые условия

$$
\begin{gathered}
y^{\prime}\left(U^{\prime} H^{\prime}+H U\right) y=0, \quad y \triangleq \Phi\left(t, t_{0} \mid \bar{A}\right) x_{0}, \quad H \triangleq D \bar{K}_{\star}, \quad U \triangleq \delta C_{\star}, \\
y^{\prime}\left(C^{\prime} V^{\prime} D^{\prime}+D V C\right) y=0, \quad D \triangleq L B+C_{\star}^{\prime} \bar{K}_{\star}^{\prime} R, \quad V=\delta \bar{K}_{\star} \in C[\mathcal{C}] .
\end{gathered}
$$

Положим $U=H^{\prime}$. Ввиду $y \neq 0$ из (4.9) будет $H H^{\prime}=0$, что возможно лишь при $H=0$, т. е. (4.9) эквивалентно условию (4.4) первой задачи. Возьмем $V=d^{\prime} c^{\prime}, c \triangleq C y, d \triangleq y^{\prime} D$, при которых $(4.10)$ есть $2\left(c^{\prime} c\right)\left(d^{\prime} d\right)=$ 
$=0$. Отсюда для выполнения (4.10) необходимо раздельное или совместное выполнение уравнений

$$
\begin{gathered}
C f_{0}={ }_{l} 0^{1}\left(f_{0}=\Phi\left(t, t_{0} \mid \bar{A}\right) \alpha_{0},\left\|x_{0}\right\| \alpha_{0} \triangleq x_{0},\left\|x_{0}\right\| C f_{0} \triangleq c\right), \\
D^{\prime} f_{0}={ }_{r} 0^{1} \quad\left(\left\|x_{0}\right\| f_{0}^{\prime} D \triangleq d\right) .
\end{gathered}
$$

Поэтому экстремальная пара $C_{*}, \bar{K}$ * второй задачи определяется либо системой (4.4), (4.11), либо (4.4), (4.12), или их пересечением - системой $(4.4),(4.11),(4.12)$. Можно показать, что в первом подслучае решение $(4.4),(4.11)$ имеет вид

$$
C_{*}=U P\left[f_{0}^{\prime}\right], \widetilde{K}_{*} \triangleq \bar{K}_{*} C_{*}=R^{-1}\left(W^{\prime}-{ }_{r} 1^{r}\right) B^{\prime} L, W \bar{K}_{*}={ }_{r} 0^{l},
$$

где $C[\mathcal{G}] \ni_{l} U^{n},{ }_{r} W^{r}, \quad U(t), W(t)-$ произвольные матрицы, $P_{[F]}={ }_{n} 1^{n}-F^{\prime}\left(F F^{\prime}\right)^{-1} F$.

Если возьмем $\forall \bar{K}_{*}(t)$ полного ранга, то $W=V_{r} P^{r}\left[\bar{K}_{*}^{\prime}\right], \forall_{r} V^{r} \in C[\mathcal{G}]$. Во втором подслучае (4.12) эквивалентно уравнению

$$
D=P\left[f_{0}^{\prime}\right] S \quad\left(\forall n S^{r}(t) \in C[\mathcal{E}]\right),
$$

из которого находим эффективную матрицу

$$
\widetilde{K}_{\star} \triangleq \bar{K}_{\star} C_{\star}=R^{-1}\left(S^{\prime} P\left[f_{0}^{\prime}\right]-B^{\prime} L\right) .
$$

Подстановкой (4.14) в (4.4) получим уравнение на $\bar{K}_{*}$ вида

$$
P\left[f_{0}^{\prime}\right] S \bar{K}_{\star}={ }_{n} 0^{l} \quad\left(P\left[f_{0}^{\prime}\right]={ }_{n} 1^{n}-f_{0}\left(f_{0}^{\prime} f_{0}\right)^{-1} f_{0}^{\prime}\right) .
$$

Таким образом, во втором подслучае экстремальная пара $C_{*}, \bar{K}_{*}$ должна быть решением совместной системы (4.14), (4.15), содержащей произвольную непрерывную матрицу $S$ порядка $(n \times \boldsymbol{r})$.

Автор благодарит О. Ваарманна, М. Қагановича, В. Ольмана и И. Петерсена за обсуждение результатов работы и полезные замечания.

\section{ЛИТЕРА Т У А}

1. Aoki, M. Joint Automat. Control. Conf. Preprint Papers. New York, 178-179 (1967).

2. Kleinman, D. L., Athans, M. IEEE Trans. Automat. Contr., 13, 150-153 (1968).

3. Ульм С. Изв. АН ЭССР. Физ. Матем., 19, № 2, 150-151 (1970).

4. Ульм С. Изв. АН ЭССР. Физ. Матем., 20, № 1, 3-7 (1971).

5. Aoki, M. In: Optimization Methods for Large-Scale Systems, 5. Aggregation. New York, McGraw Hill Book Company, 1971, 191-232.

6. Ульм С. Ю. Автоматика и телемеханика, № 5, 27-32 (1972).

7. Кейс И. Изв. АН ЭССР. Физ. Матем., 33, № 2, 201-205 (1984).

8. Athans, M., Falb, P. Optimal Control. New York, 1968, 654-676.

9. Бублик Б.' Н., Кириченко Н. Ф. Основы теории управления. Киев, «Вища школа», $1975,82-113,192-194$.

10. Первозванский А. А., Гайцгори В. Г. Декомпозиция, агрегирование и приближенная оптимизация. М., «Наука», 1979, 154-244.

Институт кибернетики

Академии наук Эстонской ССР
Поступила в редакцию 2/VII 1985 


\section{MITTESTATSIONAARSETE MITMEMOOTMELISTE SUSTEEMIDE REGULAATORITE SUBOPTIMAALNE SUNTEES LINEAARSEL AGREGEERIMISMEETODIL}

On vaadeldud ruutoptimaalsusfunktsionaaliga mitmemõôtmelise mittestatsionaarse lineaarse süsteemi juhtimisülesannet. Kolme Kleinman-Athansi ja Ulmi tüüpi kriteeriumi alusel on uuritud agregeerimis- ja võimendusmaatriksi ning ligikaudsete suboptimaalsete lineaarsete regulaatorite määramise võimalust. On saadud vajalikud ekstreemumtingimused agregeerimis- ja vōimendusmaatriksitele ja esitatud suboptimaalsed regulaatorid, mis vastavad kolmele uuritud variandile.

\section{KEIS}

\section{ON SUBOPTIMAL CONTROL SYNTHESIS IN THE LARGE-SCALE LINEAR SYSTEM VIA AGGREGATION METHOD}

The control problem of the large-scale linear dynamic system (1.1) governed by the quadratic performance criterion of quality (1.2), is considered in the paper.

The concept of aggregation is applied here in order to account for the possibility of the incomplete state feedback and/or the demand to develop computationally more efficient procedure in constructing partly optimal linear controls.

With this aim the problem of the gain and aggregation matrix optimal choice relevant for three suboptimality criteria (see $(2.1),(3.6),(4.1)$ ) is investigated.

As a result, necessary conditions adequate to the considered in sect. $2-4$ variants, are obtained for extremal matrixes in (2.3)-(2.5); (3.8), (3.9); (4.4), (4.13)-(4.15). Hence, linear suboptimal controls associated with efficient matrixes (2.4), (3.9), (4.14) are derived to advance the investigations of $M$. Aoki, $M$. Athans and S. Ulm. 